Programa Nacional de Asistencia Ventilatoria No Invasiva.

${ }^{2}$ Departamento de Pediatría y Cirugía Infantil Sur, Facultad de Medicina, Universidad de Chile, Hospital Exequiel González Cortés. Santiago de Chile. ${ }^{3}$ Consultorio Laurita Vicuña, El Bosque, Santiago de Chile. ${ }^{4}$ Unidad de Cuidados Especiales, Instituto Nacional de Rehabilitación, Pedro Aguirre Cerda. Santiago de Chile. ${ }^{5}$ Departamento de Kinesiología, Red Salud UC. Santiago de Chile. aKinesiólogo

Recibido el 28 de febrero de 2011, aceptado el 18 de abril de 2012

Correspondencia a: Dr. Daniel Zenteno A Av. José Arrieta 5969, Peñalolén, Santiago Fono: 0056-09-8180678 E-mail: danielzenteno@ gmail.com

\section{Valores de referencia del test de marcha de seis minutos en niños sanos}

\author{
DARWIN GATICA ${ }^{1, \mathrm{a}}$, HOMERO PUPPO ${ }^{2, \mathrm{a}}$, GREGORY VILLARROEL ${ }^{1, \mathrm{a}}$, \\ IVÁN SAN MARTÍN ${ }^{3, a}$, ROSSANA LAGOS 5 ,a, JUAN JOSÉ MONTECINO \\ CRISTIAN LARA ${ }^{3, a}$, DANIEL ZENTENO ${ }^{1,2,4}$

\section{Reference values for the 6-minutes walking test in healthy Chilean children}

Background: The 6-minutes walking test (6WT) is the ideal submaximal test for the evaluation and follow-up of patients with chronic respiratory diseases. There are no reference values ( $R V)$ for Chilean children using the American Thoracic Society guidelines. Aim: To generate 6WT reference values for Chilean children aged 6 to 14 years. Material and Methods: 6MW was evaluated in 192 healthy children (100 women) aged between 6 and 14 years. The test was carried out in a $30 \mathrm{~m}$ long indoor flat surface. Children also answered a survey about health problems and their weight and height were recorded. Results: The distance walked by women and men was $596.5 \pm 57$ and $625 \pm 59.7 m$ respectively $(p<0.05)$. There was a significant correlation between the distance walked and height $(r=0.58)$, age $(r=0.56)$, weight $(r=0.54)$ and reserve heart rate $(r=0.21)$. Conclusions: These results can be used as reference values for the 6WT in Chilean children aged 6 to 14 years. They are similar to those reported abroad.

(Rev Med Chile 2012; 140: 1014-1021).

Key words: Reference values; Respiratory functions tests.
$\mathrm{E}$ 1 test de marcha de seis minutos (TM6) es una herramienta cada vez más utilizada en la práctica clínica que evalúa la capacidad para realizar ejercicio físico de un sujeto, midiendo la distancia máxima que puede caminar durante 6 min. Se considera una prueba sub-máxima, debido a que provoca un estrés fisiológico en los sistemas cardiorespiratorio y muscular en condiciones de demanda aeróbica, por tanto, es un buen indicador de la capacidad para realizar ejercicio $^{1}$. Es una prueba sencilla, bien tolerada y representativa de las actividades de la vida diaria y que no requiere de equipo sofisticado para su realización ${ }^{2,3}$. Además, cumple con criterios de validez y confiabilidad tanto en niños sanos ${ }^{4-6}$, como en niños con patologías cardiopulmonares ${ }^{7,8}$. En el año 2002, la Sociedad Americana del Tórax (ATS) publicó una recomendación oficial que establece las pautas para su aplicación. Dentro de éstas se describen: el propósito, indicaciones, limitaciones, contraindicaciones, seguridad y aspectos técnicos específicos ${ }^{3}$. La creciente utilización del TM6 en la práctica clínica ha llevado a la realización de diversos estudios para unificar criterios en la aplicación del test y establecer valores de referencia en personas sanas que faciliten la comparación con pacientes que presenten patologías, principalmente de carácter crónico $^{5-15}$. En población adulta existen múltiples estudios publicados que han permitido establecer valores normales ${ }^{11-14}$. En pediatría ha sido utilizado en niños sanos ${ }^{4-6,9,10,15}$, en pacientes con fibrosis quística ${ }^{7,8}$, bronquiolitis obliterante ${ }^{16}$, cardiopatías y trasplantes cardiacos y/o pulmonares, así como también, en intervenciones terapéuticas ${ }^{17}$. Sin embargo, aún sigue siendo poco utilizado en población pediátrica y la falta de valores de referencia ha dificultado su interpretación. En Chile existe un trabajo publicado en el 
año 2001 por Escobar'; ellos propusieron valores esperados de distancia caminada de 294 niños de dos colegios de la Región Metropolitana (RM) de edad entre 6 y 14 años, obteniendo valores según edad y sexo. Este estudio ha sido de bastante utilidad en nuestro medio, sin embargo, actualmente existen algunos aspectos que cuestionan su empleo. Dentro de estos, destaca que los valores obtenidos son muy altos al ser comparados con los obtenidos de estudios internacionales ${ }^{5,6,9}$.

Debido a la escasez de datos referenciales en nuestro medio, nos propusimos evaluar a una población de niños sanos entre 6 y 14 años, de dos colegios de la RM, con la finalidad de establecer valores de referencia de distancia recorrida en 6 min utilizando un protocolo estandarizado, según guías ATS vigentes y posteriormente, comparar los datos obtenidos con la información existente en la literatura.

\section{Pacientes y Método}

Este estudio corresponde a una investigación no experimental de tipo transversal descriptivo y correlacional. La muestra correspondió a 192 niños sanos (100 mujeres), con edades comprendidas entre 6 y 14 años, que asisten a dos colegios de la RM (comuna de Maipú y Santiago) durante los meses de octubre a diciembre de 2009. Los niños incluidos cursaban desde primero a octavo básico y fueron seleccionados a través de una encuesta de estado de salud confeccionada según estudios internacionales y adaptada para ser aplicable al grupo que fue evaluado. Esta encuesta fue entregada y completada por los padres de cada niño, lo cual permitió detectar patologías respiratorias, musculoesqueléticas u otras, tanto agudas como crónicas, uso de medicamentos ocasionales y permanentes. No ingresaron al estudio niños con encuestas incompletas, índice de masa corporal (IMC) alterado, patologías agudas dentro de las últimas tres semanas, alteraciones cardiovasculares o cualquier otra patología crónica, niños con antecedentes de hospitalización en los tres últimos meses, historia de tabaquismo activo, consumo de medicamentos en forma permanente, sintomatología respiratoria y los que no tenían el consentimiento informado firmado por sus padres. El TM6 se realizó en un espacio cerrado, de superficie plana, de 30 metros de longitud, en cuyos extremos se instalaron dos conos reflectantes para señalar el límite de recorrido permitiendo que los niños giraran alrededor de ellos. En el trayecto se instalaron sillas para eventuales detenciones y marcas visibles cada 3 metros para facilitar el registro de la distancia recorrida. La prueba fue realizada por dos kinesiólogos entre 8:00 y 13:00 $\mathrm{h}$ utilizando las frases de incentivo planteadas en el protocolo estandarizado por la ATS. En una ficha diseñada especialmente para la prueba se registraron datos personales, peso, talla, además de una pregunta simple dirigida a pesquisar consumo de tabaco. Los niños utilizaron calzado y ropa adecuada. Luego de un reposo de 10 min, se explicó en forma clara e individual las características de la prueba. Se registraron antes del TM6, al final y a los 3 min posteriores frecuencia respiratoria (FR), frecuencia cardiaca (FC) y saturación de oxígeno $\left(\mathrm{SpO}_{2}\right)$ medida por un oxímetro de pulso transcutáneo (Nonin 9500; Nonin Medical, Inc., Minneapolis, EUA), sensación subjetiva de fatiga de extremidades inferiores (SSF) y de disnea (SSD) utilizando la escala modificada de Borg. Luego se calculó el porcentaje de la frecuencia cardiaca de reserva (FCR) utilizando la formula de Karvonen $^{18}$ (\%FCR $=$ FC actividad-FC reposo / FC máxima teórica-FC reposo). Para el análisis se utilizó estadística descriptiva para presentar los datos como media y desviación estándar. Para comparar rendimiento por género y grupo de edad se utilizó la prueba de Wilcoxon para muestras independientes. Para el grupo total $(\mathrm{n}=192)$ se utilizó análisis de varianza. Se aplicó la prueba $\mathrm{R}$ de Pearson para analizar posibles correlaciones entre las variables independientes y distancia recorrida. Usando el modelo de regresión lineal múltiple se determinó la mejor combinación de variables para estimar la distancia recorrida y establecer la ecuación respectiva. Para el análisis de los resultados, se utilizó el software SPSS 15.0, para Windows. En todos los casos los resultados fueron considerados significativos cuando $\mathrm{p}<0,05$.

\section{Resultados}

Todos los sujetos completaron la prueba sin complicaciones o efectos adversos. En la Tabla 1 se describen las características antropométricas del grupo separados por edad y sexo. Sólo existen diferencias significativas en la variable talla en los grupos de 8,13 y 14 años $(\mathrm{p}<0,05)$ en los cuales 
Test de marcha de 6 minutos en niños - D. Gatica et al

Tabla 1. Características antropométricas

\begin{tabular}{|lccccccccc|}
\hline & $\mathbf{6}$ años & $\mathbf{7}$ años & $\mathbf{8}$ años & $\mathbf{9}$ años & $\mathbf{1 0}$ años & $\mathbf{1 1}$ años & $\mathbf{1 2}$ años & $\mathbf{1 3}$ años & $\mathbf{1 4}$ años \\
& $\mathrm{F}$ & $\mathrm{F}$ & $\mathrm{F}$ & $\mathrm{F}$ & $\mathrm{F}$ & $\mathrm{F}$ & $\mathrm{F}$ & $\mathrm{F}$ & $\mathrm{F}$ \\
& $(\mathrm{n}=11)$ & $(\mathrm{n}=9)$ & $(\mathrm{n}=12)$ & $(\mathrm{n}=12)$ & $(\mathrm{n}=10)$ & $(\mathrm{n}=8)$ & $(\mathrm{n}=12)$ & $(\mathrm{n}=15)$ & $(\mathrm{n}=11)$ \\
& $1,20 \pm$ & $1,26 \pm$ & $1,27 \pm$ & $1,37 \pm$ & $1,44 \pm$ & $1,47 \pm$ & $1,57 \pm$ & $1,59 \pm$ & $1,60 \pm$ \\
Talla (m) & 0,04 & 0,05 & $0,04^{* * *}$ & 0,04 & 0,06 & 0,02 & 0,07 & $0,06^{* * *}$ & $0,04^{* * *}$ \\
& $22,83 \pm$ & $23,62 \pm$ & $27,35 \pm$ & $31,05 \pm$ & $36,85 \pm$ & $39,39 \pm$ & $46,54 \pm$ & $50,95 \pm$ & $52,4 \pm$ \\
Peso (kg) & 1,90 & 2,48 & 2,38 & 4,07 & 5,13 & 4,20 & 6,01 & 5,75 & 4,68 \\
& $15,77 \pm$ & $15,48 \pm$ & $16,93 \pm$ & $16,53 \pm$ & $17,83 \pm$ & $18,33 \pm$ & $18,88 \pm$ & $20,11 \pm$ & $20,47 \pm$ \\
IMC (peso/talla $)$ & 0,83 & 1,16 & 0,84 & 1,73 & 1,62 & 1,64 & 1,76 & 1,66 & 1,37 \\
& $M$ & $M$ & $M$ & $M$ & $M$ & $M$ & $M$ & $M$ & $M$ \\
& $(n=9)$ & $(n=7)$ & $(n=11)$ & $(n=6)$ & $(n=12)$ & $(n=9)$ & $(n=14)$ & $(n=12)$ & $(n=12)$ \\
& $1,21 \pm$ & $1,29 \pm$ & $1,31 \pm$ & $1,41 \pm$ & $1,40 \pm$ & $1,45 \pm$ & $1,54 \pm$ & $1,65 \pm$ & $1,66 \pm$ \\
Talla (m) & 0,04 & 0,07 & $0,05^{* * *}$ & 0,06 & 0,06 & 0,05 & 0,08 & $0,07 * * *$ & $0,05 * * * *$ \\
& $22,79 \pm$ & $26,07 \pm$ & $28,13 \pm$ & $32 \pm$ & $33,73 \pm$ & $37,78 \pm$ & $46,49 \pm$ & $53,93 \pm$ & $53,5 \pm$ \\
Peso (kg) & 1,57 & 2,37 & 3,38 & 2,88 & 4,41 & 3,9 & 6,07 & 4,47 & 5,81 \\
& $15,62 \pm$ & $15,73 \pm$ & $16,15 \pm$ & $16,17 \pm$ & $17,03 \pm$ & $17,99 \pm$ & $19,48 \pm$ & $19,87 \pm$ & $19,49 \pm$ \\
IMC (peso/talla $\left.{ }^{*}\right)$ & 0,70 & 0,80 & 1,05 & 1,14 & 1,28 & 1,32 & 1,03 & 1,28 & 1,83 \\
\hline
\end{tabular}

F: Femenino; M: Masculino; IMC: Índice de Masa Corporal; m: metros; kg: kilogramos. ${ }^{* * *} \mathrm{p}<0,05$.

Tabla 2. Parámetros iniciales y finales en el TM6

\begin{tabular}{|c|c|c|c|c|c|c|}
\hline \multirow[b]{2}{*}{ Variables } & \multicolumn{3}{|c|}{ Mujeres } & \multicolumn{3}{|c|}{ Hombres } \\
\hline & Inicio & Final & \% Diferencia & Inicio & Final & $\%$ Diferencia \\
\hline FC & $84 \pm 10$ & $127 \pm 17$ & $32,9 \pm 0,9 \%$ & $84 \pm 10$ & $124 \pm 17$ & $31,5 \pm 0,9$ \\
\hline$F R$ & $17 \pm 3$ & $25 \pm 4$ & $29,6 \pm 1 \% * * *$ & $18 \pm 3$ & $25 \pm 5$ & $26,3 \pm 1,1^{* * *}$ \\
\hline $\mathrm{SpO}_{2}$ & $98 \pm 1$ & $97 \pm 1$ & $0,6 \pm 0,2 \%$ & $98 \pm 1$ & $97 \pm 1$ & $0,6 \pm 0,1$ \\
\hline SSF & $1 \pm 1$ & $4 \pm 3$ & $3,5 \pm 0,2^{* * *}$ & $1 \pm 1$ & $3 \pm 3$ & $2,6 \pm 0,2^{* * *}$ \\
\hline SSD & $1 \pm 1$ & $3 \pm 3$ & $2,7 \pm 0,2$ & $1 \pm 1$ & $3 \pm 2$ & $2,6 \pm 0,2$ \\
\hline FCR \% & \multicolumn{3}{|c|}{$34,1 \pm 12,3$} & \multicolumn{3}{|c|}{$31,9 \pm 12$} \\
\hline
\end{tabular}

FC: Frecuencia cardiaca ; FR: Frecuencia respiratoria ; $\mathrm{SpO}_{2}$ : Saturación de oxígeno; SSF: Sensación subjetiva de fatiga; SSD: Sensación subjetiva de disnea; FCR: Frecuencia cardiaca de reserva. ${ }^{* * *} p<0,05$.

los hombres resultaban más altos que las mujeres. En la Tabla 2 se describen las variables evaluadas al inicio, al final y la diferencia entre ellas, separadas por género. Se produjo un aumento en la FC, FR, SSF Y SSD y la SpO, se mantuvo en rangos normales en ambos grupos. Sólo existieron diferencias significativas en el porcentaje de aumento de la FR $(29,6 \pm 1 \%$ mujeres; $26,3 \pm 1,1 \%$ hombres; $\mathrm{p}=0,03)$, y la SSF $(3,5 \pm 0,2$ mujeres; $2,6 \pm 0,2$ hombres; $\mathrm{p}=0,006)$. La evaluación realizada a los 3 min posteriores a la finalización del TM6 marcó un regreso a los niveles basales de todos los pará- metros. La distancia recorrida en promedio para el grupo fue de $610,4 \pm 57 \mathrm{~m}(596,5 \pm 50,7 \mathrm{~m}$ en mujeres; 625,6 \pm 59,7 $\mathrm{m}$ en hombres; diferencia de 29,1 m; p < 0,05). En la Tabla 3 y Figura 1 se expresan los valores de distancia recorrida por edad y sexo. En el análisis por rango etáreo se encontró que en la mayoría de los grupos los hombres recorrieron una distancia mayor que las mujeres, exceptuando en el grupo de 6 años. Sólo existen diferencias estadísticas significativas en el grupo de 8 y 13 años, en donde los varones caminaban $49,3 \mathrm{~m}(\mathrm{p}=0,0489<0,05)$ y $42,5 \mathrm{~m}$ 
Test de marcha de 6 minutos en niños - D. Gatica et al

Tabla 3. Distancia recorrida en el TM6 según edad y sexo

\begin{tabular}{|lccccccccc|}
\hline & $\mathbf{6}$ años & $\mathbf{7}$ años & $\mathbf{8}$ años & $\mathbf{9}$ años & $\mathbf{1 0}$ años & $\mathbf{1 1}$ años & $\mathbf{1 2}$ años & $\mathbf{1 3}$ años & $\mathbf{1 4}$ años \\
Mujeres & $562,5 \pm$ & $568,2 \pm$ & $556,5 \pm$ & $575,7 \pm$ & $585,7 \pm$ & $606,7 \pm$ & $629,4 \pm$ & $631,4 \pm$ & $638,5 \pm$ \\
& 39,1 & 31,6 & $48,9 * * *$ & 53,2 & 28,7 & 60,3 & 20,3 & $50,2^{* * *}$ & 20,9 \\
Hombres & $562 \pm$ & $597,11 \pm$ & $605,8 \pm$ & $611,4 \pm$ & $618,7 \pm$ & $608,7 \pm$ & $636,1 \pm$ & $673,9 \pm$ & $674,3 \pm$ \\
& 43,1 & 33,6 & $62,2 * * *$ & 47 & 67,5 & 35,9 & 47,3 & $45^{* * *}$ & 54,2 \\
Total & $562,3 \pm$ & $580,9 \pm$ & $580,1 \pm$ & $587,6 \pm$ & $603,7 \pm$ & $607,8 \pm$ & $633 \pm$ & $650,3 \pm$ & $657,2 \pm$ \\
& 39,8 & 34,7 & 59,9 & 52,8 & 55 & 47,3 & 36,8 & 51,8 & 44,7 \\
\hline
\end{tabular}

$* * * p<0,05$.

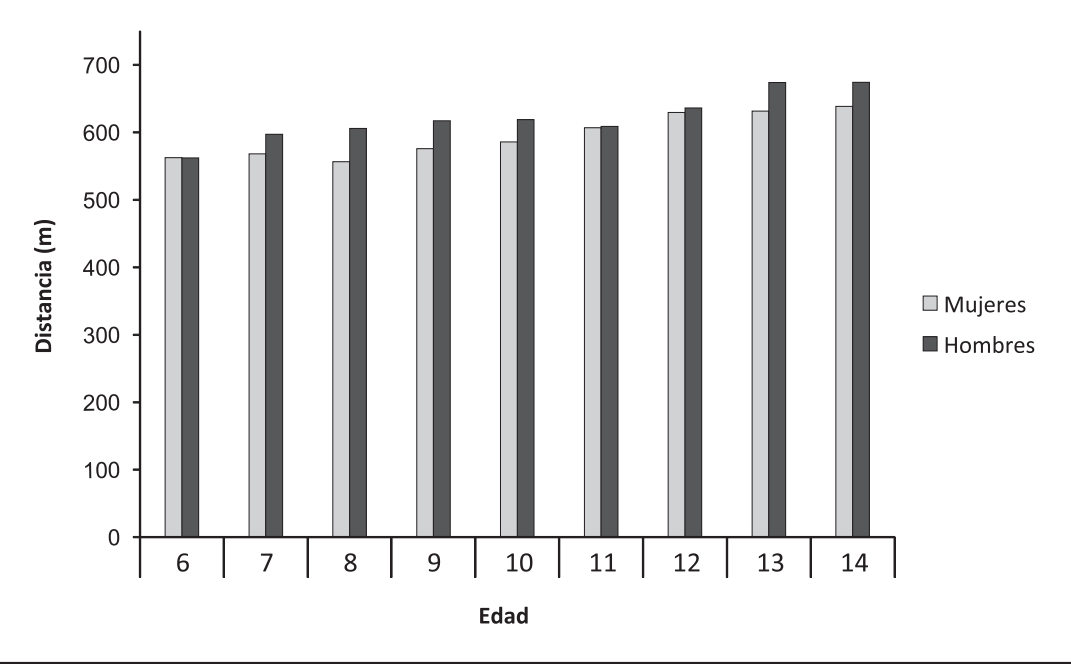

Figura 1. Distancia recorrida por edad y género.

más que las niñas $(\mathrm{p}=0,0381<0,05)$ respectivamente. Utilizando la prueba estadística $\mathrm{R}$ de Pearson encontramos una correlación positiva entre distancia y talla $\mathrm{r}=0,577$; edad $\mathrm{r}=0,556$, peso $r=0,535,(p<0,0001)$ y FCR $r=0,207$ $(\mathrm{p}=0,004)$. Al aplicar el modelo de regresión lineal múltiple se determinó que la edad, peso, talla y FCR utilizada representan el 34,4\% de la distancia recorrida y corresponde al mejor modelo para la ecuación. Debido a las diferencias entre la distancia recorrida entre hombres y mujeres hemos establecidos 3 ecuaciones para realizar la comparación con estudios previos.

a) Ecuación para el grupo total $(\mathrm{n}=192)$

Distancia $=265,058+[227,455 *$ talla $(\mathrm{m})]+$ $[6,103 *$ edad (años) $]-[1,701 *$ peso $(\mathrm{kg})]+$ $[61,489 *$ Frecuencia cardiaca de reserva] $\mathrm{R}^{2}=34,4 \%$; Error $=46,14 \mathrm{mts}$. b) Ecuación para hombres $(\mathrm{n}=92)$

Distancia hombres $=331,404+(158,523 *$ talla $)$ $+\left(11,945^{\star}\right.$ edad $)-(2,139$ * peso $)+(70,221$ * Frecuencia cardiaca de reserva) $\mathrm{R}^{2}=29,9 \%$; Error $=49,96 \mathrm{mts}$.

c) Ecuación para mujeres $(\mathrm{n}=100)$ Distancia mujeres $=274,566+\left(208,818^{*}\right.$ talla $)$ $+(2,337$ * edad $)-(0,682$ * peso $)+\left(77,849^{\star}\right.$ Frecuencia cardiaca de reserva) $\mathrm{R}^{2}=39,6 \%$; Error $=39,43$ mts.

La correlación de nuestros datos predictivos con las ecuaciones de Priesnitz, Geiger y Li se presenta en la Tabla 4 (Figuras 2-4). Encontramos una fuerte correlación con los valores de Priesnitz para el grupo total $(r=0,996, \mathrm{p}<0,0001) \mathrm{y}$ Geiger, (mujeres $r=0,911$, hombres $r=0,951$; $p$ $<0,0001)$. Con los valores de Li la correlación fue menor pero sigue siendo considerable (mujeres $r$ 
Tabla 4. Correlación entre valores predicitvos

\begin{tabular}{|c|c|c|c|}
\hline & Población & $\mathbf{n}$ & $\mathbf{r}$ \\
\hline Priesnitz & Brasil & $\begin{array}{c}188 \\
\text { (92 hombres) }\end{array}$ & $0,996^{* *}$ \\
\hline \multirow[t]{2}{*}{ Geiger } & Austria & $\begin{array}{c}280 \\
\text { (hombres) }\end{array}$ & 0,951 ** \\
\hline & & $\begin{array}{c}248 \\
\text { (mujeres) }\end{array}$ & 0,911 ** \\
\hline \multirow[t]{2}{*}{ Li } & China & $\begin{array}{c}805 \\
\text { (hombres) }\end{array}$ & $0,798 * *$ \\
\hline & & $\begin{array}{c}640 \\
\text { (mujeres) }\end{array}$ & $0,663^{* *}$ \\
\hline
\end{tabular}

** La correlación es significativa al nivel de 0,01.

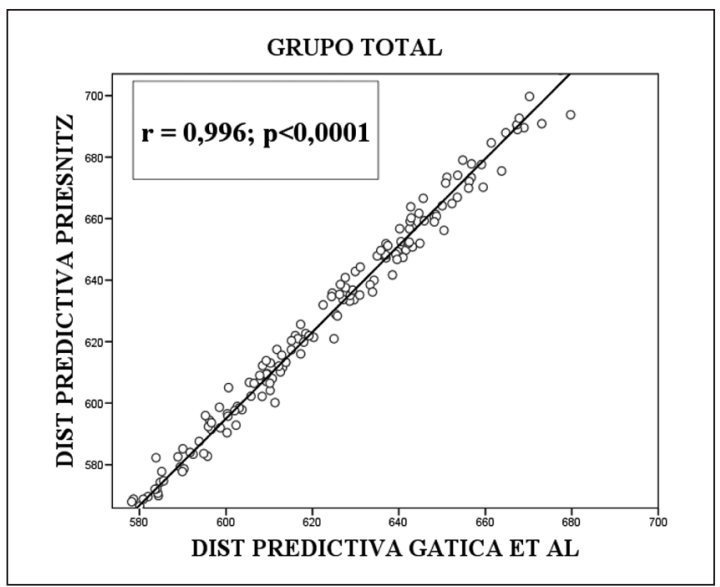

Figura 2. Correlación valores predictivos de Priesnitz.

B

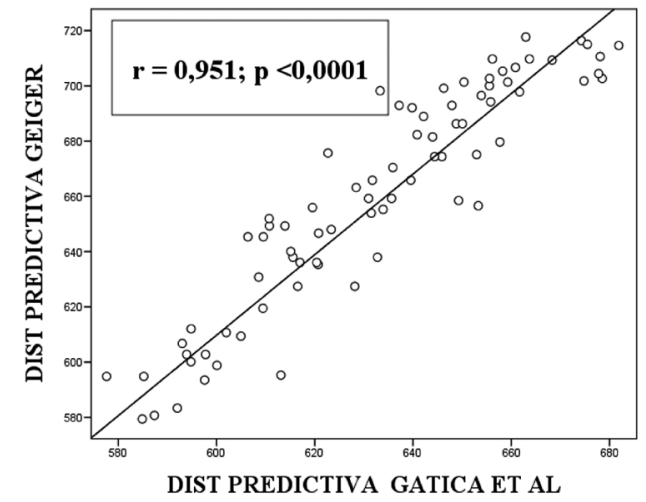

Figura 3 A y B. Correlación valores predictivos de Geiger.

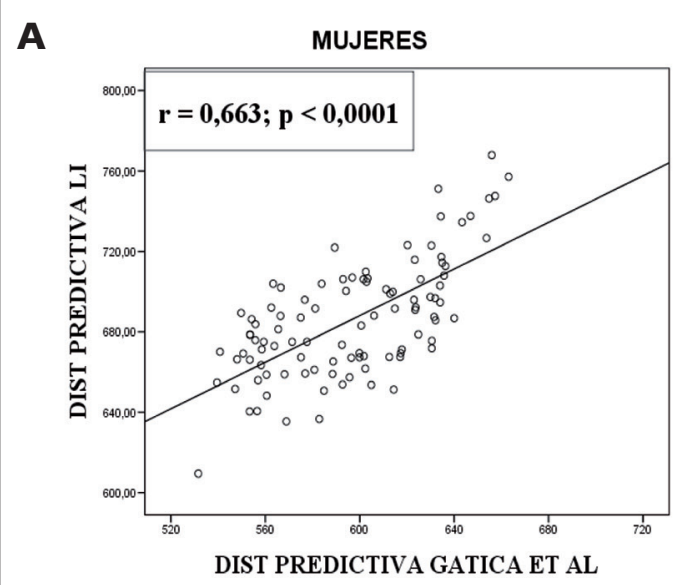

B

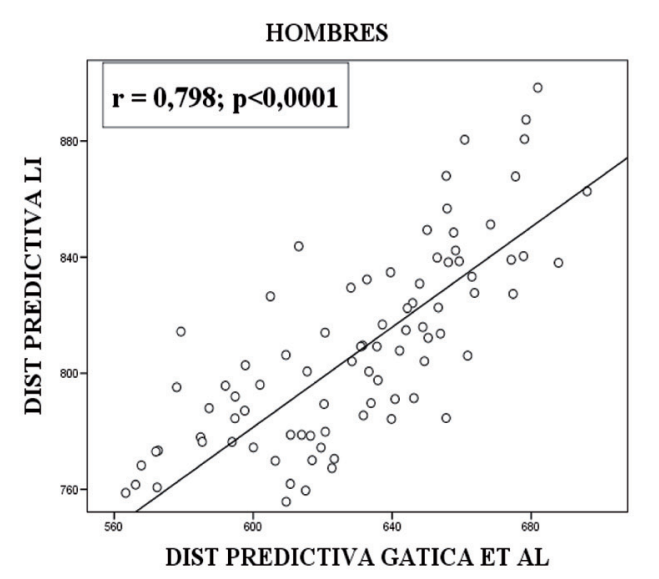

Figura 4 A y B. Correlación valores predictivos de Li. 
Test de marcha de 6 minutos en niños - D. Gatica et al

Tabla 5. Comparación de distancia recorrida con valores obtenidos por Escobar y cols

\begin{tabular}{|llcccc|}
\hline Edad & Sexo & $\begin{array}{c}\text { Distancia Escobar y } \\
\text { cols (2001) } \\
\text { (media } \pm \text { desv. estándar) }\end{array}$ & $\begin{array}{c}\text { Distancia Gatica y } \\
\text { cols (2010) } \\
\text { (media } \pm \text { desv. estándar) }\end{array}$ & $\begin{array}{c}\text { Diferencia } \\
\text { distancia } \\
\text { (metros) }\end{array}$ & \% Diferencia \\
\hline $6-8$ años & Femenino & $652,8 \pm 62,1$ & $561,9 \pm 40,3$ & 90,9 & 13,93 \\
& Masculino & $650,1 \pm 63,9$ & $588,9 \pm 52,0$ & 61,1 & 9,4 \\
\hline 9-11 años & Femenino & $722,9 \pm 55$ & $599,3 \pm 46,4$ & 123,6 & 17,09 \\
& Masculino & $722,4 \pm 62,8$ & $613,7 \pm 52,6$ & 108,7 & 15,04 \\
\hline $12-14$ años & Femenino & $737,2 \pm 57,1$ & $634,4 \pm 40,0$ & 102,8 & 13,94 \\
& Masculino & $809,9 \pm 77$ & $660,1 \pm 51,1$ & 149,8 & 18,49 \\
\hline
\end{tabular}

$=0,663$, hombres $\mathrm{r}=0,798 ; \mathrm{p}<0,0001)$. A nivel nacional tenemos el estudio de Escobar ${ }^{9}$ del año 2001. Para realizar la comparación con sus resultados agrupamos nuestros valores de acuerdo a 3 categorías de edad como se presenta en la Tabla 5. En todos los grupos nuestros resultados de distancia fueron inferiores tanto en hombres como en mujeres. En el grupo de 6 a 8 años existía una diferencia que va desde 9,4 a $13,93 \%$; en el grupo de 8 a 11 años de 15,04 a 17,09\% y por último en el grupo de 12 a 14 años la diferencia se extendió desde el 13,94 a 18,49\%.

\section{Discusión}

El presente estudio proporciona ecuaciones y valores de referencia para predecir la distancia recorrida en el TM6, para niñas y niños sanos residentes en la RM según estándares ATS. En Chile existe poca evidencia de su aplicación en población pediátrica y tampoco contamos con valores de referencia fiables y aceptados por las sociedades científicas nacionales. Nuestra motivación está dirigida a aumentar la utilización de esta prueba para establecer de forma sencilla el perfil de capacidad física de un paciente con patología cardiopulmonar y poder realizar una comparación fiable en el tiempo de la tolerancia al ejercicio. En el grupo total se observó que los varones recorren 29,1 m más que las mujeres. Al realizar el análisis por edad sólo encontramos diferencias significativas en los de 8 y 13 años lo que se podría explicar a que en dichos subgrupos la talla de los varones es estadísticamente superior, con lo cual lograrían un mejor rendimiento debido a un largo de zancada mayor. Encontramos una correlación positiva entre distancia recorrida y talla, edad, peso y porcentaje de utilización de la FCR lo que está en concordancia con los resultados presentados por otros autores $5^{5,6,9,10,15}$. Al realizar el análisis con el modelo de regresión lineal múltiple se encontró que las variables seleccionadas representan el 34,4\% de la distancia, es probable que factores como la motivación, condición física, coordinación y habilidades motoras de los participantes estuvieran relacionados en un mejor rendimiento. Los valores de referencia existentes en pediatría son escasos y relativamente recientes. Geiger ${ }^{4}$ determinó valores de normalidad en una población de 456 niños sanos caucásicos según edad y sexo entre 3 y 18 años, logrando estandarizar la distancia caminada desde temprana edad. Destaca una diferencia entre géneros donde los varones caminan una mayor distancia que las niñas. Dentro del mismo año $\mathrm{Li}^{9}$ en un estudio de 1.445 niños chinos sanos estandarizó valores de normalidad estableciendo la talla como factor determinante y creando un nomograma de distancia caminada entre los percentiles 25 y 75 . En el año 2009 Priesnitz $^{6}$ estableció valores de referencia en 188 niños sanos de 6 a 12 años en una población brasileña. Estos tres estudios presentan ecuaciones de referencia de distancia recorrida basadas en la talla, peso, edad y diferencia de FC antes y después del TM6. Al comparar nuestros resultados predictivos con estos estudios hemos encontrado una alta correlación con Priesnitz ${ }^{6}$ y Geiger $^{5}$ y una correlación más baja pero considerable con los valores de $\mathrm{Li}^{9}$. La alta correlación se debe probablemente a similitudes en grupos de edades, características antropométricas y la aplicación de 
un mismo régimen de estímulo (incentivos $\mathrm{ATS}^{2}$ ), la menor correlación con los valores de $\mathrm{Li}^{9}$ podría establecerse por diferencias raciales, grupo etáreo y posiblemente también a variables antropométricas. A nivel nacional el contraste con el estudio publicado por Escobar ${ }^{10}$ resulta más notorio. Si bien ambos fueron realizados en la RM y las edades eran similares, la aplicación de un protocolo diferente conlleva a diferencias importantes en los valores obtenidos de distancia recorrida por ambos grupos (Tabla 5). En el transcurso del test el uso establecido de las frases de estímulo para continuar el ejercicio que el evaluador emite adquieren gran importancia, debido a que su intensidad, entusiasmo y frecuencia representa importantes diferencias en los resultados ${ }^{3,20}$. En este sentido pudieran darse diferencias cuando dos evaluadores realizan la prueba, sin embargo, cuando se utiliza un protocolo estandarizado la confiabilidad interobservador es alta $^{4}$ (coeficiente de correlación intraclase 0,94$)$. Al evaluar la confiabilidad test-retest no existen diferencias significativas entre dos TM6 separados por 18 días $^{4}$ y por $30 \mathrm{~min}^{6}$ con una diferencia media de $15 \mathrm{~m}$ y $8,78 \mathrm{~m}$ respectivamente. Desde este punto de vista parece razonable descartar la posibilidad de un efecto de aprendizaje en una prueba de práctica, razón por la cual nuestro grupo decidió realizar un solo test. Por otro lado, además de la representación fisiológica de rendimiento aeróbico expresado en las distintas variables evaluadas posterior al TM6, se realizó el cálculo del porcentaje de utilización de la FCR utilizando la formula de Karvonen que establece un mejor perfil de rendimiento físico dentro de una prueba submáxima. Mediante esta fórmula se obtiene una estimación válida de la carga cardiovascular. En condiciones controladas, el porcentaje de FCR que se emplea al realizar un trabajo físico está fuertemente asociado al porcentaje del consumo de oxígeno de reserva empleado ${ }^{19}$, de tal forma que al medir la primera se obtiene una estimación cercana del costo energético de dicha actividad. Por último, el conocimiento del rendimiento observado en la aplicación del protocolo de la ATS para el TM6 a niños sanos, complementa la interpretación de los datos obtenidos en sujetos con disfunción del movimiento, enfermedades cardiacas o respiratorias crónicas. En relación con la incidencia clínica, estos datos derivados de la marcha expresada en rendimiento físico aeróbico, nos permite determinar el grado de proximidad de los pacientes a los rangos de normalidad. En síntesis, el protocolo de ATS para el TM6, cumple con los requisitos necesarios para estresar el sistema cardiorrespiratorios del sujeto por medio de una prueba funcional eficiente que hace entrega de manera rápida y oportuna de datos confiables, válidos y útiles para la retroalimentación en la práctica clínica y aplicables con facilidad en distintos centros de nuestro país con personal entrenado ${ }^{1}$. Los resultados presentados en este estudio establecen valores de referencia de distancia recorrida en el TM6 en niños chilenos sanos de 6 a 14 años, determinando las principales variables que inciden en un mejor rendimiento. Se generan ecuaciones de referencia como una medida útil para establecer una adecuada tolerancia al ejercicio físico.

Agradecimientos: Los autores de este trabajo agradecen enormemente el apoyo brindado por los colegios Ozanam, de Santiago y al colegio Luis Gandarillas, de Maipú; a sus directores, profesores y alumnos por su buena disposición para con nuestro grupo.

\section{Referencias}

1. Zenteno D, Puppo H, González R, Kogan R. Test de marcha de 6 minutos en pediatría. Neumol Pediát 2007; 2 (2): 109-14.

2. Solway S, Brooks D, Lacasse Y, Thomas S. A qualitative systemic overview of the measurement properties of functional walk tests used in the cardiorespiratory domain. Chest 2001; 119: 256-70.

3. ATS statement: guidelines for the six-minute walk test. Am J Respir Crit Care Med 2002; 166: 111-7.

4. Li A M, Yin J, Yu C C, Tsang T, So H K, Wong E, et al. The six-minute walk test in healthy children: reliability and validity. Eur Respir J 2005; 25: 1057-60.

5. Geiger R, Strasak A, Treml B, Gasser K, Kleinsasser A, Fischer V, et al. Six-Minute Walk Test in Children and Adolescents. J Pediatr 2007; 150: 395-9.

6. Priesnitz C, Horak G, Da Silva C, Viapiana G, Posteraro C, Tetelbom R, et al. Reference Values for the 6-min Walk Test in Healthy Children Aged 6-12 Years. Pediatr Pulmonol 2009; 44: 1174-9.

7. Gulmans V, Van Veldhoven N, De Meer K, Helders P. The six-minute walking test in children with cystic fibrosis: reliability and validity. Pediatr Pulmonol 1996; 22: 85-9.

8. Cunha M, Rozov T, Caitano de Oliveira R, Jardim J. 
Test de marcha de 6 minutos en niños - D. Gatica et al

Six-Minute Walk Test in Children and Adolescents With Cystic Fibrosis. Pediatr Pulmonol 2006; 41: 618-22.

9. Li A M, Yin J, Au J, So H, Tsang T, Wong E, et al. Standard reference for the 6-minute walk test in healthy children aged 7 to 16 years. Am J Respir Crit Care Med 2007; 176: 174-80.

10. Escobar M, López A, Veliz C, Crisóstomo S, Pinochet R. Test de Marcha en 6 minutos en niños Chilenos. Kinesiología 2001; 62: 16-20.

11. Enright P, Sherrill D. Reference equations for the sixminute walk in healthy adults. Am J Respir Crit Care Med 1998; 158: 1384-7.

12. Troosters T, Gosselink R, Decramer M. Six minute walking distance in healthy elderly subjects. Eur Respir J 1999; 14: 270-74.

13. Gibbons W, Fruchter N, Sloan S, Levy R. Reference values for a multiple repetition 6-minutes walking test in healthy subjects older than 20 years. J Cardiopulm rehabil 2001; 21: 87-93.

14. Casanova C, López M, Marín J, De Torres J, Casas A, Montes De Oca M. Six minute walk distance in a multicenter study of healthy subjects aged 40-80 years in Spain and South America. Am J Respir Crit Care Med
2007; 175: A955.

15. Lammers AE, Hislop AA, Flynn Y, Haworth SG. The 6-minute walk test: normal values for children of 4-11 years of age. Arch Dis Child 2008; 93: 464-8.

16. Zenteno D, Puppo H, González R, Pavón D, Vera R, Torres R, et al. Test de Marcha de Seis Minutos en Niños con Bronquiolitis Obliterante Postviral. Correlación con Espirometría. Rev Chil Enfer Respir 2008; 24: 15-9.

17. Moalla W, Gauthier R, Maingourd Y, Ahmaidi S. SixMinute Walking Test to Assess Exercise Tolerance and Cardiorespiratory Responses During Training Program in Children With Congenital Heart Disease. Int J Sports Med 2005; 26: 756-62.

18. Strath S, Swartz AM, Basset D, O'Brien L, King A, Ainswortz B. Evaluation of heart rate as a method for assessing moderate intensity physical activity. Med Sci Sports Exerc 2000; 32: 465-70.

19. Dalleck LC, Kravitz L. Relationship Between \%Heart Rate Reserve and \% $\mathrm{VO}_{2}$ Reserve during Elliptical Crosstrainer Exercise. J Sports Sci Med 2006; 5: 662-71.

20. Guyatt GH, Pugsley SO, Sullivan MJ, Thompson PJ, Berman LB, Jones NL, et al. Effect of encouragement on walking test perfomance. Thorax 1984; 39: 818-22. 